\title{
Discordance between aPTT and anti-factor Xa levels and implications in patients receiving intravenous unfractionated heparin therapy
}

\author{
Akwasi Opoku BA, Kenneth Iwuji MD, Brendon Clough PharmD, Jacqueline Le PharmD, \\ McKenzie Moore PharmD, Chelsi Simmons PharmD, Bridget Hyde PharmD
}

\begin{abstract}
Heparin, one of the world's oldest anticoagulation medications, accelerates the rate of inhibition of previously activated clotting factors. It is most often used in the prophylaxis and treatment of thromboembolic disorders and complications associated with atrial fibrillation. The two most common ways to monitor plasma heparin levels and anticoagulation therapy are the activated partial thromboplastin time (aPTT) and anti-factor Xa assay (anti-Xa). This article assesses the performance of aPTT and anti-Xa monitoring protocols and analyzes the discordance between aPTT and anti-Xa levels and its clinical implications in patients receiving intravenous heparin therapy.
\end{abstract}

Keywords: unfractionated heparin, heparin, aPTT, anti-factor Xa, discordance, clotting

\section{BACKGROUND}

Heparin, discovered in 1916 and first approved by the FDA in 1939, is one of the oldest anticoagulant medications still in use today. Twenty years later (1959), the FDA approved Warfarin. These two medications were the mainstays of anticoagulation therapy until the 1980s when low-molecular weight heparin (LMWH) was developed. Direct oral anticoagulants, dabigatran (Pradaxa) and rivaroxaban (Xeralto), were approved in 2010 followed by apixaban (Eliquis) in 2012 and edoxaban (Savaysa) in 2015. ${ }^{1}$

Heparin does not act directly on coagulation factors. Rather, it acts indirectly by binding to antithrombin III which induces a conformational change, making the active site of antithrombin more accessible to thrombin (factor II) and factor Xa. Antithrombin then acts as a

Corresponding author: Kenneth Iwuji

Contact Information: Kenneth.Iwuji@ttuhsc.edu

DOI: 10.12746/swrccc.v7i30.559 suicide inhibitor of activated clotting factors. The conformational change causes increased rates of factor $\mathrm{Xa}$ inhibition but does not affect the rate of thrombin inhibition. However, the heparin molecule itself acts as a catalyst for the binding of antithrombin to thrombin with its ability to bind to both molecules simultaneously. Thus, heparin inhibits factors Xa and Ila in a 1:1 ratio. ${ }^{2,3}$

Beyond its use in prophylaxis and treatment of thromboembolic disorders and complications associated with atrial fibrillation, heparin is indicated for the prevention of clotting in cardiac surgery and as an anticoagulant for dialysis procedures and extracorporeal circulation. ${ }^{4}$ Heparin possesses several off-label uses, including an alternative to other anticoagulants to prevent the formation of thrombus during percutaneous coronary intervention, treatment of patients with acute coronary syndrome undergoing invasive or ischemiaguided strategy, and an adjunctive option to fibrinolytic therapy in patients presenting with ST-elevation myocardial infarction. Heparin can also be used off-label in superficial vein thrombosis, cardioversion of patients with atrial fibrillation, non-bacterial thrombotic endocarditis, cerebral venous sinus thrombosis, and acute arterial emboli. $^{5}$ 


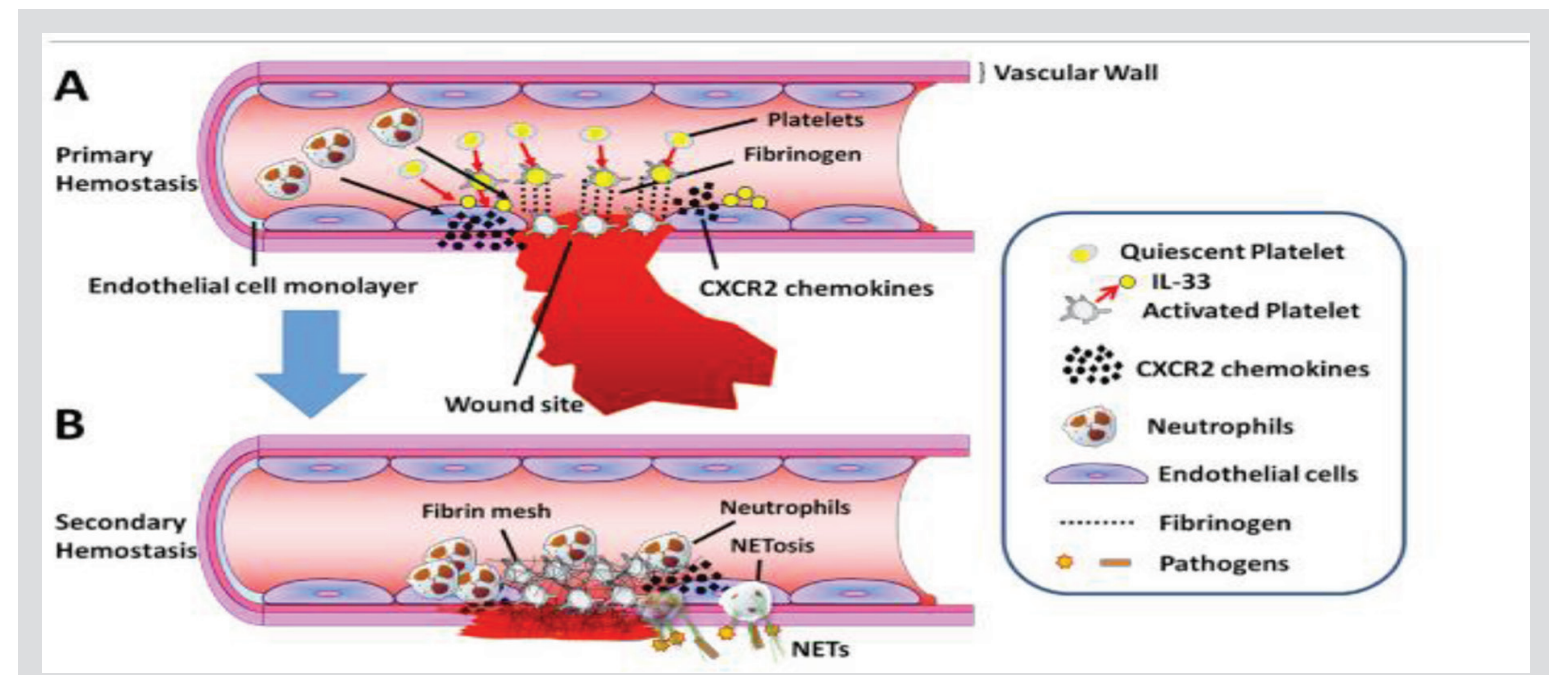

Figure 1. Steps of primary hemostasis: platelet adhesion, activation, and aggregation.

(Image from Takeda, T., Morita, H., Saito, H., Matsumoto, K., \& Matsuda, A. Recent advances in understanding the roles of blood platelets in the pathogenesis of allergic inflammation and bronchial asthma. Allergology International 2018;67(3):326-333. $)^{8}$

\section{HEMOSTASIS - THE BODY'S TUG OF WAR}

The human body maintains a dynamic balance between thrombogenic and fibrinolytic systems to prevent both thrombosis and hemorrhage. Hemostasis takes place when pro-thrombotic components of the body overcome the body's natural anti-thrombogenic components, either as result of increased thrombogenic activity or decreased anti-thrombogenic activity. ${ }^{6}$

Hemostasis begins with the formation of a hemostatic plug (Figure 1) that occurs in a series of steps: platelet adhesion, platelet activation, and platelet aggregation. The endothelial cells lining blood vessels possess anti-thrombotic properties. However, vascular injury can expose the subendothelial layer which is highly thrombogenic and contains materials, such as collagen and von Willebrand factor (VWF), that increase platelet adhesion. ${ }^{6,7}$ After vascular injury, VWF binds to glycoprotein $\mathrm{Ib}$ in the platelet membrane, thus allowing platelets to adhere to the subendothelial layer. Adherence of platelets activates them, causing them to secrete the contents of their alpha and dense granules. Release of calcium attracts various coagulation factors; adenosine diphosphate (ADP) and thromboxane A2 (TxA2) recruit more platelets and further activate those platelets, leading to the formation of the primary platelet plug (Figure 1). Once the plug has bridged the gap caused by the vascular injury, adjacent endothelial cells release prostacyclin to prevent further expansion of the plug. A chain of reactions, termed the coagulation cascade, then ensues to stabilize the platelet plug and form a fibrin clot (Figure 2).

\section{CoAgulation CASCADE - TWO HIGHWAYS LEADING TO THE SAME DESTINATION}

The coagulation cascade involves two pathways that merge at a common point to form a stable fibrin clot. The intrinsic pathway includes factors XII, XI, IX, and VIII. The extrinsic pathway includes factors III (tissue factor) and VII. The intrinsic and extrinsic pathway merge at the activation factor $X$ and form the common pathway, which includes factors X, V, II (prothrombin). If the coagulation cascade is disrupted, particularly in the common pathway, fibrin formation is affected, which impedes clot formation. ${ }^{2}$

Virchow's triad, postulated by Rudolph Virchow in 1856, consists of three factors that promote the 


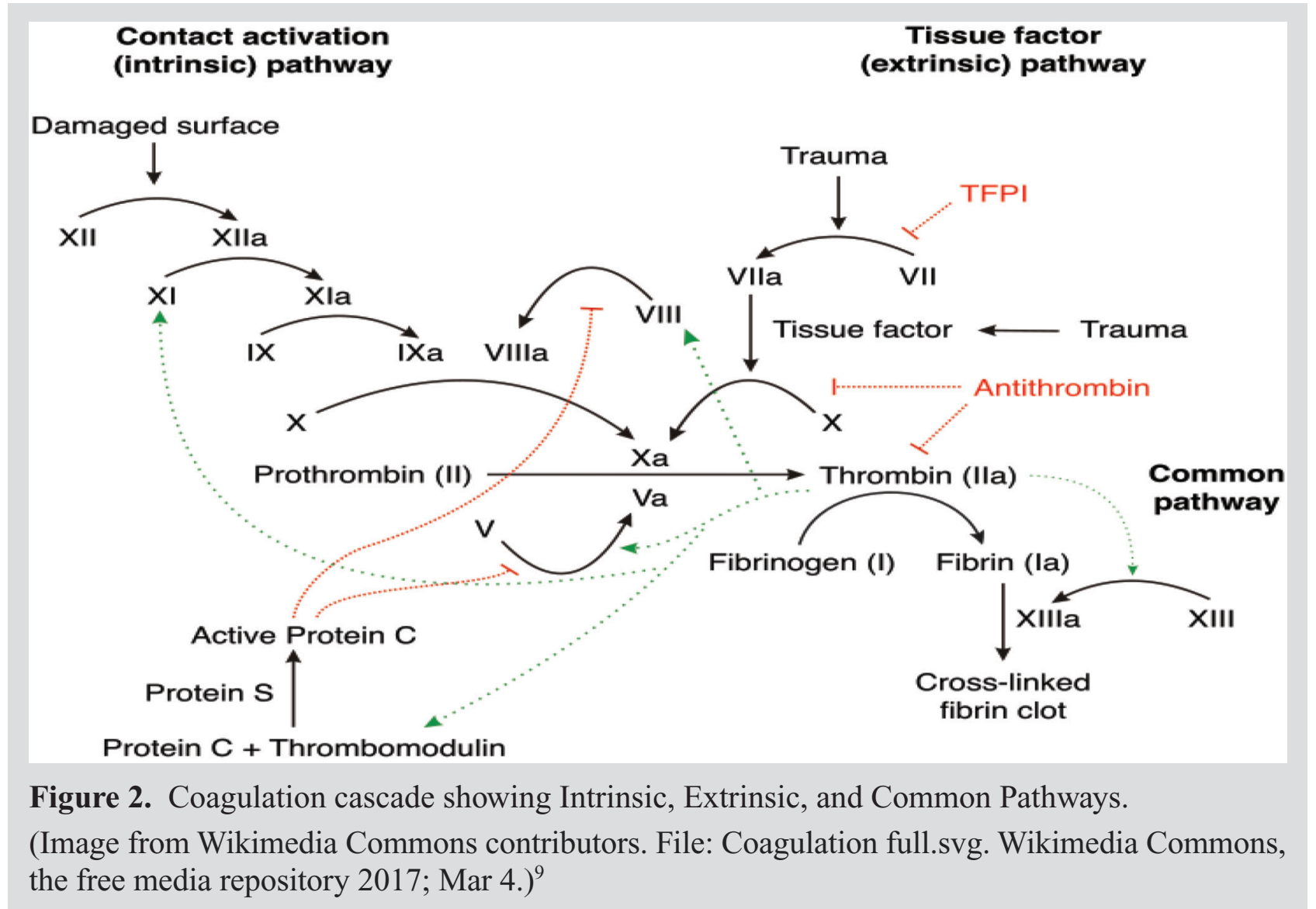

formation of thrombosis: blood flow stasis, hypercoagulability, and endothelial injury. ${ }^{10}$ Blood flow stasis occurs in the context of congestive heart failure and lengthy periods of immobilization in prolonged bed rest and long-distance travel. A hypercoagulable state can result from protein $\mathrm{C}$ or $\mathrm{S}$ deficiencies, factor $\mathrm{V}$ Leiden mutation, antiphospholipid syndrome, heparininduced thrombocytopenia, obesity, smoking, or oral contraceptive use. ${ }^{11}$ Endothelial injury exposes the sub-endothelial layer to the contents of the bloodstream, which leads to the formation of a hemostatic plug. $^{10}$

\section{THERAPEUTIC MONITORING}

Activated partial thromboplastin time (aPTT) is a test that characterizes the coagulation of blood. It is most commonly used to monitor the therapeutic effects of heparin. In 1972, aPTT monitoring was tested by Basu et al in a prospective clinical trial that involved 234 patients being treated with continuous IV heparin. The objective was to investigate the relationship between aPTT and recurrent venous thromboembolism (VTE) or bleeding. Heparin dosing was maintained between 0.5-2.5 times the control level of normal plasma, which was 39-41 seconds for that laboratory. The aPTT was measured at least once daily, and episodes of bleeding or recurrent venous thromboembolism (VTE) were recorded. The five patients who suffered recurrent VTE had lower aPTT (49 sec) than patients who did not have recurrent VTE (66 sec). This difference in aPTT was statistically significant. Patients who had aPTT $<50$ seconds for two consecutive days had a $7 \%$ risk of VTE recurrence with risk increasing to $21 \%$ at three consecutive days. The investigators concluded that 
the risk of recurrent VTE can be reduced by maintaining aPTT at 1.5-2.5 times the control level at all times. $^{12}$

Unlike unfractionated heparin, which exerts its effects on both factor II and factor Xa, low-molecular weight heparin (LMWH) predominantly acts on factor Xa. As such, LMWH activity is monitored using serum anti-factor Xa (anti-Xa) levels instead of aPTT. The target anti-Xa ranges for therapeutic doses of LMWHs has been well established by many studies. For example, peak anti-Xa levels of 1.0-2.0 and 0.6$1.0 \mathrm{IU} / \mathrm{mL}$ have been identified as therapeutic for daily and bi-daily administration of subcutaneous enoxaparin, respectively. ${ }^{13}$ In contrast, a target range for prophylactic doses of LMWH is not well established due to lack of supporting evidence. A review article of published studies by Wei et al concluded that a reasonable anti-Xa range for VTE prophylaxis with LMWH might be $0.2-0.5 \mathrm{IU} / \mathrm{mL} .{ }^{13}$

\section{LABORATORY MEASUREMENTS OF APTT AND ANTI-FACTOR XA LEVELS}

Activated partial thromboplastin time (aPTT) is an "in vitro" coagulation test commonly used to assess the intrinsic and common pathways of the coagulation cascade. Whole blood, when added to a glass tube, begins to clot because the glass activates factor XII which initiates the intrinsic pathway. To prevent blood from clotting in the tube, a calcium chelating agent such as citrate is first placed into the tube before the addition of blood. Platelet substitutes such as silica and factor XII activators, which are negatively charged phospholipids, are then added to the blood, and the sample is incubated. The sample is then recalcified. The time it takes for the blood to form a stable clot is measured in seconds and termed the aPTT. A whole blood sample in a glass tube typically takes between 4 to 8 minutes to clot. Recalcified plasma typically clots in 2 to 4 minutes. However, recalcified plasma with phospholipids and platelet substitutes clots in approximately 26 to 33 seconds. ${ }^{13,14,15}$

Variables that affect the measurement of aPTT include the type of reagent used, length of incubation, and method of measuring the clot formation. ${ }^{14,15}$ Patient disease states (liver disease, factor VIII, IX, and XII deficiencies, lupus anticoagulant syndrome, antiphospholipid syndromes) also affect aPTT levels. Therefore, aPTT levels from different laboratories cannot be compared directly. Variations can even exist within the same laboratory using the same reagent. This accounts for the highly variable aPTT nomograms that exist in different institutions. However, for heparin monitoring, it is recommended that each laboratory establish aPTT and anti-Xa ranges that correspond to 0.2 to $0.4 \mathrm{unit} / \mathrm{mL}$ by protamine titration. ${ }^{16}$

Anti-Xa level is becoming the gold standard for monitoring therapeutic levels for patient receiving intravenous unfractionated heparin. Anti-Xa level is determined by a chromogenic assay performed by spectrophotometry. A patient's plasma is added to a reagent factor $\mathrm{Xa}$, and the activity of factor $\mathrm{Xa}$ is measured using an artificial factor $X$ a substrate that releases a colored compound when cleaved. The presence of heparin in a patient's blood sample inhibits some of the reagent factor $\mathrm{Xa}$. The uninhibited or residual factor Xa reacts with the chromogenic substrate, which is quantified by spectrophotometry absorbance. The residual factor Xa activity is inversely proportional to the concentration of heparin in the blood sample. This test is more expensive than the cost of measuring an aPTT level. ${ }^{17,18}$

Dosing nomograms are used by many hospitals to adjust heparin dosages and shorten delays in achieving and maintaining therapeutic aPTT or anti-Xa levels. ${ }^{19,20}$ There are numerous nomograms currently in use with variations in administration instructions regarding heparin bolus and rate changes. These variations may be due to the multiple variables that affect aPTT that were previously mentioned. While these nomograms may be generalizable, a standardized dosing nomogram protocol is needed.

A randomized controlled trial by Raschke et al showed that a weight-based heparin nomogram is safer and more effective than one based on standard practice. The study included 150 patients requiring IV heparin for treatment of venous or arterial thromboembolism and for unstable angina. Patients were randomly assigned to a weight-based 
nomogram ( 80 units $/ \mathrm{kg}$ bolus plus 8 units $/ \mathrm{kg} / \mathrm{hr}$ infusion) or standard care nomogram (5000-unit bolus plus 1000 units/hr infusion). The aPTT was then measured every six hours. A therapeutic range of 1.5-2.3 times the control was used. In the weightbased group, 60 out of 62 patients $(97 \%)$ exceeded the therapeutic threshold of aPTT > 1.5 within 24 hours, compared with 37 out of 48 patients (77\%) in the standard care group. Additionally, recurrent thromboembolism was more frequent in the standard care group with a relative risk ratio of $5: 1$. The one incidence of bleeding complication occurred in the standard care group. ${ }^{20}$

\section{LITERATURE REVIEW}

The most common methods for monitoring the infusion of unfractionated heparin are the anti-Xa assay and aPTT. Many studies have compared the performance of anti-Xa and aPTT protocols. A singlecenter prospective cohort study by Samuel et al involved 85 patients receiving heparin infusion for the management of ischemic stroke and treatment of VTE. ${ }^{21}$ Forty-eight patients were assigned to the aPTT group and 37 patients to the anti-Xa group. The investigators of the study concluded that the anti-Xa assay might be a better monitoring tool than aPTT based on the period of time the values were within the targeted therapeutic range. Ten percent of patients in the aPTT group ( 5 total patients) reached therapeutic range compared to $57 \%$ of patients in the anti-Xa group (21 total patients). This statistically significant difference also led to less blood drawing in the anti-Xa group. Second, patients in the anti-Xa group reached therapeutic range sooner than those in the aPTT group. The amount of time needed to reach therapeutic range was 22 hours for the aPTT group and 15 hours for the anti-Xa group. Third, the study showed that paired anti-Xa and aPTT values were discordant $57 \%$ of the time by analyzing 234 paired measurements from 37 patients. Fifty-two percent of data pairs (68) had high aPTT values with subtherapeutic anti-Xa measurements, and 54\% (53) had high aPTT values with therapeutic anti-Xa measurements. Two patients suffered bleeding complications as a result of the discordant pattern.
Both patients had supratherapeutic aPTT values and either a subtherapeutic or therapeutic anti-Xa values. ${ }^{21}$

A retrospective, single center, cohort study by Vandiver et al compared anti-Xa and aPTT monitoring of IV heparin in the treatment of pulmonary embolism (PE) and DVT. The records of 186 patients (88 managed by anti-Xa assay-based protocol and 98 managed by aPTT-based protocol) were assessed to determine how often monitoring values were within target therapeutic range. ${ }^{22}$ The study showed that the use of anti-Xa assay-based monitoring protocol resulted in statistically significantly higher percentage of within-range monitoring values than the aPTT protocol $(69 \%$ vs $41 \%)$. In addition, patients in the anti-Xa group reached therapeutic levels sooner and received fewer dose adjustments than those in the aPTT group.22

A randomized, controlled trial by Levine et al included 131 patients with acute DVT, PE, or axillary vein thrombosis who had received at least 35,000 units of continuous heparin infusion during the previous 24 hours. The patients' heparin therapy was monitored by either aPTT or anti-Xa assay. Three out of 65 patients in the anti-Xa group (4.6\%) experienced recurrent VTE compared with four out of 66 patients in the aPTT group $(6.1 \%) .{ }^{16}$ Four $(6.1 \%)$ bleeding events occurred in the aPTT group, but the anti-Xa group had a single episode (1.5\%). Patients in the aPTT group needed statistically significant more heparin than those in the anti-Xa group during the bridging period before the initiation of warfarin. Furthermore, the daily mean aPTT values were subtherapeutic for the anti-Xa group but within therapeutic range for the aPTT group. However, the daily mean anti-Xa levels were within target therapeutic range for both groups. ${ }^{16}$

Arachchillage et al compared the validity of the aPTT monitoring with that of the anti-Xa assay in 3,543 samples from 475 patients receiving unfractionated heparin. Included in the study were 165 infants, 60 children between 1-15 years, and 250 adults. The overall concordance of aPTT and anti-Xa values was poor. In adult patients, concordance $(51.8 \%)$ and discordance $(48.4 \%)$ were nearly 
equal. Among adult patients who had therapeutic anti-Xa levels, only $38 \%$ had therapeutic aPTT values; $56 \%$ had subtherapeutic aPTT values, and $6 \%$ had supra-therapeutic aPTT values. Among infants whose anti-Xa were within therapeutic range, the majority of samples had supratherapeutic levels of APTT. This discordance could potentially result in under anticoagulation in infants or bleeding complications in adult patients. ${ }^{23}$

\section{Conclusion}

The aPTT is often used to monitor therapy with unfractionated heparin, but it may not provide an accurate measurement of the amount of heparin present. Factors that contribute to the variability of aPTT measurements include the type of reagent used, length of incubation, and method of measuring the clot formation. Because the anti-factor Xa assay is less susceptible to confounding variables, it may be a better assay for the monitoring of heparin therapy. There is increasing evidence that shows that monitoring IV unfractionated heparin with anti-Xa assays attains therapeutic anticoagulation faster, maintains therapeutic range longer, and demands fewer blood draws and adjustments in dosages.

Article citation: Opoku A, Iwuji K, Clough B, Le J, Moore M, Simmons C, Hyde B. Discordance between aPTT and anti-factor Xa levels and implications in patients receiving intravenous unfractionated heparin therapy. The Southwest Respiratory and Critical Care Chronicles 2019;7(30):12-18

From: Department of Internal Medicine $(\mathrm{KI})$, The School of Medicine (AO, BC), and the School of Pharmacy (JL, MM, CS, BH), Texas Tech University Health Sciences Center, Lubbock, Texas

Submitted: $5 / 20 / 2019$

Accepted: 4/7/2019

Reviewer: Victor Test MD

Conflicts of interest: none

This work is licensed under a Creative Commons Attribution-ShareAlike 4.0 International License.

\section{REFERENCES}

1. Royston D. Anticoagulant and antiplatelet therapy. In: Pharmacology and Physiology for Anesthesia: Foundations and Clinical Application 2013:643-667.

2. Harter K, Levine M, Henderson SO. Anticoagulation drug therapy: a review. West J Emerg Med 2015;16(1):11-17.

3. Hogg K, Weitz JI. Blood Coagulation and Anticoagulant, Fibrinolytic, and Antiplatelet Drugs. In: Brunton LL, Hilal-Dandan R, Knollmann BC. eds. Goodman \& Gilman's: The Pharmacological Basis of Therapeutics, 13e New York, NY: McGraw-Hill; http://accesspharmacy.mhmedical.com. ezproxy.ttuhsc.edu/content.aspx? bookid=2189 \&sectionid=170271546. Accessed November 15, 2018.

4. Heparin. In: Lexi-Drugs [database online]. Hudson, Ohio: Wolters Kluwer Health. Updated periodically. Accessed November 26, 2018.

5. Guyatt GH, Akl EA, Crowther M, et al. Executive Summary: antithrombotic therapy and prevention of thrombosis, 9th ed: American College of Chest Physicians Evidence-Based Clinical Practice Guidelines. Chest 2012;141(2 Suppl):7S-47S.

6. Palta S, Saroa R, Palta A. Overview of the coagulation system. Indian J Anaesth 2014;58(5):515-23.

7. Hawiger J. Formation and regulation of platelet and fibrin hemostatic plug. Hum Pathol 1987;18(2):111-122.

8. Takeda T, Morita H, Saito H, et al. A. Recent advances in understanding the roles of blood platelets in the pathogenesis of allergic inflammation and bronchial asthma. Allergology International 2018;67(3):326-333.

9. Wikimedia Commons contributors. File: Coagulation full.svg. Wikimedia Commons, the free media repository; 2017 Mar 4.

10. Kyrle PA, Eichinger S. Is Virchow's triad complete? Blood 2009;114:1138-1139.

11. Schafer AI. The hypercoagulable states. Ann Intern Med 1985;102:814-828.

12. Basu D, Gallus A, Hirsh J, et al. Cade J. A prospective study of the value of monitoring heparin treatment with the activated partial thromboplastin time. N Engl J Med 1972;287(7): 324-327.

13. Wei MY, Ward SM. The anti-Factor Xa range for low molecular weight heparin thromboprophylaxis: Hematol Rep 2015; 7(4):5844.

14. Ignjatovic V. Activated partial thromboplastin time. Methods Mol Biol 2013;992:111-120.

15. Adcock DM, Bethel MA, Macy PA. Coagulation Handbook. Austin, TX: Esoterix, Inc.; 2006.

16. Levine MN, Hirsh J, Gent M, et al. A randomized trial comparing activated thromboplastin time with heparin assay in patients with acute venous thromboembolism requiring large daily doses of heparin. Arch Intern Med 1994;154(1):49-56. 
17. Newall F. Anti-factor Xa (anti-Xa) assay. Methods Mol Biol 2013;992:265-272.

18. Aguilera J, Yousef H, Beltran-Melgarejo D, et al. Clinical scenarios for discordant anti-Xa. Advances in Hematology 2016, Article ID 4054806.

19. Cruickshank MK, Levine MN, Hirsh J, et al. A standard heparin nomogram for the management of heparin therapy. Arch Intern Med 1991;151(2):333-7.

20. Raschke RA, Reilly BM, Guidry JR, et al. The weight-based heparin dosing nomogram compared with a "standard care" nomogram. A randomized controlled trial. Ann Intern Med 1993;119(9):874-881.
21. Samuel S, Allison TA, Sharaf S, et al. Antifactor Xa levels vs. activated partial thromboplastin time for monitoring unfractionated heparin. A pilot study. J Clin Pharm Ther 2016;5: 499-502.

22. Vandiver JW, Vondracek TG. A comparative trial of anti-factor Xa levels versus the activated partial thromboplastin time for heparin monitoring. Hosp Pract 2013;41(2):16-24.

23. Arachchillage DRJ, Kamani F, Deplano S, et al. Should we abandon the APTT for monitoring unfractionated heparin? Throb Res 2017;157:157-61. 